\title{
LA EVALUACIÓN DEL DESEMPEÑO EN EL CONTEXTO DE CLASES REMOTAS
}

\author{
Fredy Yesid Arenas Torres* ${ }^{*}$ Edgar Balaguera Ascencio**, Jairo Andrés Coral Campaña ${ }^{* * *}$, \\ Luis Orlando Herrera García ${ }^{* * * *}$, José Hermes Martínez Saavedra ${ }^{* * * * *}$ \\ Universidad Santo Tomás \\ Alberto Montalvo Castro******, Yeimmy Katherine Serrano León ${ }^{* * * * * * * *}$ \\ Universidad Agustiniana \\ https://doi.org/10.14718/9789585133846.2021.3
}

* Magíster en Educación Matemática del Centro de Investigación y Formación en Educación (CIFE) de la Universidad de los Andes y Licenciado en Educación Básica con Énfasis en Matemáticas de la Universidad Distrital Francisco José de Caldas.

https://orcid.org/0000-0002-4293-785X

* Doctor en Educación Matemática. Docente e investigador del Departamento de Ciencias Básicas de la Universidad Santo Tomás.

https://orcid.org/0000-0002-9501-8320

Magíster en Materiales y Procesos, Físico. Líder de Investigación del Departamento de Ciencias Básicas de la Universidad Santo Tomás. https://orcid.org/0000-0003-2075-9009

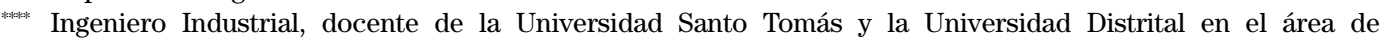
Programación y Métodos Numéricos. https://orcid.org/0000-0001-6857-3188

Magíster en educación, Licenciado en Matemáticas de la Universidad Distrital. Docente en la Universidad Santo Tomás y en la Universidad del Rosario. https://orcid.org/0000-0003-2924-3666

Licenciado en Matemáticas y Física, docente del Departamento de Ciencias Básicas de la Universitaria Agustiniana. https://orcid.org/my-orcid?orcid=0000-0002-6034-6981

* Cursante del Máster Universitario en Análisis y Visualización de Datos Masivos / Visual Analytics and Big Data. Especialista en Estadística y Licenciada en Matemáticas. yeimmy.serrano@uniagustiniana.edu.co 


\section{Introducción}

La pandemia generada por la COVID-19 trajo consigo cambios drásticos y repentinos en todas las actividades que a diario se realizan. En el contexto de la educación, fue necesaria la construcción e implementación de múltiples estrategias de flexibilización curricular con el fin de dar continuidad a los procesos iniciados de forma presencial durante el primer semestre del año 2020. Rápidamente, y prácticamente de un día para otro, las actividades académicas, así como los procesos de interacción en aula, migraron hacia un trabajo virtual y, en el mejor de los casos, a un entorno de mediaciones sincrónicas facilitadas por las herramientas propias de la tecnología, con todos los inconvenientes que esto conlleva, sin una capacitación previa suficiente y sin lograr que todos los actores del proceso académico se integraran activamente.

Para desarrollar los procesos de seguimiento y acompañamiento en el marco del ejercicio particular de la evaluación se requirió de la construcción sistemática de un conjunto de estrategias que permitieran superar las necesidades generadas en la nueva realidad académica.

Teniendo esto en cuenta, en el presente capítulo se reflexiona respecto al desempeño de los estudiantes en los diferentes espacios académicos administrados por los Departamentos de Ciencias Básicas en la Universidad Santo Tomás, sede Bogotá, y la Universitaria Agustiniana, considerando la observación en tres periodos diferentes: un semestre prepandemia (2019-2), un semestre de transición (2020-1), y un semestre en medio de la pandemia (2020-2).

El estudio parte de la siguiente premisa: los métodos, didácticas, entornos y plataformas virtuales - tipo Moodle, Edmodo, Classroom, entre otros- orientados hacia el desarrollo de los procesos de acompañamiento, seguimiento y medición, propios del compromiso curricular de la evaluación en estudiantes de educación superior que iniciaron su formación académica en un ambiente virtual, no responden a un cumplimiento suficiente de los resultados de aprendizaje definidos en el marco de un conjunto de competencias específicas enmarcadas en un desarrollo tipo modalidad presencial, y por tanto no pueden aplicarse automática o tácitamente sin el desarrollo de un periodo de transición y sensibilización de estas herramientas a estudiantes que iniciaron en un sistema presencial y que tuvieron que trasladarse a un entorno virtual por la emergencia sanitaria causada por la COVID-19 en el 2020, por las razones que se exponen a lo largo del documento.

En este sentido, las metas correspondientes para este estudio son: (a) identificar algunas de las problemáticas relacionadas con los procesos de evaluación en la educación superior como consecuencia de la interacción no presencial emergente en la nueva realidad académica, a partir del estudio realizado a una muestra particular de estudiantes vinculados con la Universidad Santo Tomás y la Universitaria Agustiniana; y (b) explicitar las alternativas de evaluación aplicadas a la muestra y los resultados obtenidos, para una posible implementación, en caso de mantenerse - o volver a presentarse- las condiciones de interrupción de la interacción física en sistemas presenciales, o en su aplicación en sistemas híbridos o virtuales. 


\section{Desarrollo}

Con la llegada de la COVID-19 a Colombia, el Estado promulgó normas de impacto institucional en todos los niveles que afectaron los contextos propios de la cotidianidad académica, comercial, social, política, entre otros, con el fin de generar el denominado distanciamiento social, y con ello controlar la propagación del virus. Esta respuesta fue coherente con la declaración de emergencia mundial, y fue un aspecto que afectó también de forma directa las actividades escolares (Luthra, 2020) públicas y privadas, en todos los niveles de formación —básica, media y superior-, de carácter formal o informal.

De esta manera, el sistema educativo nacional, que sin importar su carácter o nivel de formación había adelantado hasta ese entonces sus procesos dentro del contexto de la presencialidad, se vio obligado a reconsiderar sus acciones académicas, desde los beneficios extendidos de las tecnologías de la información y la implementación de los contextos híbridos posibles, en el estado de transición generado por la interrupción de la educación presencial (Vicenti, 2020).

Estas transformaciones impactaron las condiciones pedagógicas y didácticas, así como aquellas relacionadas con el seguimiento y el control de los procesos propios de la enseñanza y el aprendizaje. Como afirma Camacho (2020), en este nuevo escenario, la evaluación del proceso de aprendizaje de los estudiantes, a diferencia del Espacio Europeo de Educación Superior (EEES), busca adoptar un proceso de formación basado en las competencias de los estudiantes con el uso de metodologías mediadas por recursos educativos tales como entornos virtuales, internet, bases de datos, aplicaciones informáticas y gamificación, entre otros.

No obstante, el sistema educativo colombiano se distancia de estas características, y aunque las dos instituciones sobre las cuales se centra la reflexión poseen programas de formación superior en contextos virtuales, ninguna desarrolla a plenitud un trabajo dentro de un contexto que valide con suficiencia el uso de las tecnologías.

Ahora bien, respecto a la evaluación, entendida como una construcción particular y propia de cada una de las instituciones, que se fundamenta desde los objetivos institucionales del PEI, y desde la perspectiva de Trujillo et al. (2020), es posible encontrar cuatro escenarios concretos que representan distintas concepciones de la evaluación desde una línea institucional: (a) el primer escenario, denominado autonomía de los centros, se caracteriza por establecer una mínima toma de decisiones por parte de la administración gubernamental, por lo cual es posible que las instituciones den solución a las consideraciones metodológicas de la evaluación; (b) el segundo escenario corresponde a un ajuste del currículo, y plantea la toma de decisiones en un nivel medio, con la cual es posible la solución desde la justicia y la equidad que implican los procesos evaluativos, considerando un ámbito de actuación particular referido desde las instituciones de educación; (c) el tercer escenario, denominado evaluación positiva condicionada, se caracteriza por la toma de decisiones por parte de la administración gubernamental en relación con la evaluación del curso 2019-2020; y (d) por 
último, el cuarto escenario se denomina evaluación positiva sin condiciones, y se caracteriza por la promoción de todos los estudiantes tras pretender solucionar las dificultades a mediano plazo durante el 2020-2021.

En los dos primeros contextos la responsabilidad del proceso evaluativo se concentra en los centros educativos, los docentes, los estudiantes y sus familias, en tanto que la responsabilidad no recae en el Estado, mientras que para los escenarios evaluación positiva condicionada y no condicionada el Ministerio de Educación Nacional es el responsable directo de emitir los lineamientos generales.

En línea con Trujillo et al. (2020), tanto la Universidad Santo Tomás como la Universitaria Agustiniana corresponden significativamente con los escenarios de autonomía del centro y ajuste curricular, razón por la cual conviene identificar un conjunto de estrategias que permitan ser aplicadas en el seguimiento y la evaluación antes y durante el desarrollo de la pandemia, con el fin de asociarlas a las diferentes variaciones encontradas en los desempeños alcanzados por los estudiantes en el marco de los procesos académicos registrados en los espacios propios de los Departamentos de Ciencias Básicas.

Respecto a los aspectos definidos por la autonomía de centro y el ajuste curricular, las instituciones objeto de estudio implementaron un modelo denominado presencialidad mediada por tecnología (PMT), cuyo propósito general se centra en atender las orientaciones generales del Gobierno respecto a la prevención y no expansión de la COVID-19, y con lo cual se establece la continuidad de los procesos de enseñanza y aprendizaje a través de espacios académicos mediados exclusivamente por la tecnología.

Este modelo PMT garantiza la oferta educativa por parte de las instituciones mediante el desarrollo de actividades y recursos para el aprendizaje a través del uso de las plataformas virtuales particulares de cada institución y el uso de las sesiones presenciales sincrónicas — clases virtuales - en el horario respectivo de cada espacio académico, y es precisamente este nuevo escenario el objeto de estudio en esta investigación, ya que cualquier cambio implica ser observado, monitoreado, analizado y evaluado para lograr una categorización del fenómeno con miras en el futuro a estudios con mayor profundidad.

Con el fin de avanzar con la reflexión, se considera pertinente examinar el concepto de evaluación, del cual se promueve una diversidad de significados por la variación que ha tenido durante los últimos años.

Para inicios del siglo xx, la evaluación en la educación era considerada sinónimo de medición por el apoyo desde las ideas conductistas y positivistas en el proceso de formación, las cuales, según Casanova (2011), conciben la evaluación como el "proceso de obtención sistemática de datos que ofrece información continua acerca del modo en que se produce la enseñanza y el 
aprendizaje" (p. 84). De este modo, la evaluación se distanció de los resultados de condición memorística y se acercó estrechamente a la medición de un conjunto de resultados de aprendizaje fundamentados en el desarrollo de un grupo de competencias que se relacionan con un grupo de contenidos disciplinares, de modo sumativo, formativo, continuo y permanente, interiorizados y aplicados en contextos fenomenológicos propios de la realidad del estudiante. En este sentido, se insertó el proceso tecnológico racional al proceso educativo, y con él a la formación; no obstante, esta perspectiva fue posteriormente superada por la consideración cualitativa de la misma, y se fue trasladando este acto a un plano de la diversidad humana como es el fenómeno educativo.

Esta condición ha generado de la evaluación un campo investigativo cuya práctica se encuentra inmersa en el marco social, con prácticas complejas desde múltiples puntos de referencia y que varían según las condiciones del contexto. De este modo, tal como lo plantea Nevo (1997), la evaluación pasó a componerse de elementos técnicos y metodológicos por un lado, y éticos y morales por otro, donde estos últimos cobran un mayor nivel de importancia.

Por esta razón, la evaluación educativa corresponde a la acción de recopilar de forma sistemática la información relacionada con la naturaleza y calidad de los objetos vinculados con el proceso educativo, elementos que varían con el juicio valorativo, que no es más que la valoración propuesta por quien administra el modelo o por quienes acuerdan el parámetro.

Por otra parte, para Nevo (1997) la evaluación educativa consiste en la recogida sistemática de información referente a la naturaleza y la calidad de los objetos educativos; definición que combina la descripción con el juicio crítico, pero que hace una distinción entre ambos, ya que son de naturaleza distinta. De este modo, la descripción puede basarse en la recogida sistemática de datos, y así dar como resultado información altamente objetiva. En cambio, el juicio crítico se basa en criterios que, en la mayor parte de los casos, están determinados por valores y normas sociales.

\section{Descripción y metodología}

Este trabajo se enmarca en la investigación cuantitativa de tipo descriptivo, ya que se enfoca en el análisis y comprensión de un fenómeno social que permite dar respuesta a un problema, en el cual los participantes — que son objetos de estudio— se encuentran dentro de un entorno natural. Sabino (2014) señala que los tipos de investigación descriptivos permiten encontrar y describir características fundamentales de fenómenos en conjuntos homogéneos que, mediante la sistematización de la información y los procesos de comparación con otras fuentes, posibilitan la comprensión respecto a su estructura y comportamiento. 
Una vez delimitado el problema a estudiar, es importante — dentro del método descriptivo— la elaboración y construcción de las técnicas e instrumentos para la observación, recolección y registro de los datos. Esta fase permite tomar la mayor cantidad de detalles posibles en torno al fenómeno observado, de tal forma que al ser organizados, será más fácil procesar la información, considerando su importancia y significado. Finalmente, mediante la interpretación y análisis de la información, se logra categorizar aspectos o características fundamentales del fenómeno estudiado.

En consideración de lo anterior, el objeto de estudio de la presente investigación plantea como hipótesis general que el grado de valoración cuantitativa —enmarcada entre 1.0 y 5.0 alcanzado por los estudiantes de programas profesionales que transitaron repentinamente de un escenario presencial a uno mediado por plataformas tecnológicas —como consecuencia de la pandemia - presentan una variación significativa cuando existe un desarrollo metodológico mediado por encuentros presenciales sincrónicos en plataformas, respecto a los desempeños obtenidos en modalidad presencial regular.

Como consecuencia de la hipótesis, es posible generar la siguiente pregunta orientadora: ¿Las nuevas estrategias de evaluación y seguimiento de forma sincrónica o asincrónica —utilizando diferentes herramientas tecnológicas - han influido de manera positiva en la nota o concepto final que alcanzan los estudiantes en los espacios académicos administrados por los Departamentos de Ciencias Básicas?

Es importante aclarar que determinar si cada uno de los estudiantes ha logrado asimilar los conocimientos mínimos en función de un grupo de competencias desarrolladas no corresponde con el objeto de estudio definido para esta investigación, considerando que estos aspectos desarrollan un análisis de procesos y metodologías de aprendizaje que están por fuera de los límites del presente estudio.

Así, esta investigación permitirá evaluar algunas situaciones o características particulares de estos nuevos modelos de educación, dada la particularidad por la contingencia de la COVID-19. Teniendo en cuenta su carácter descriptivo, es de gran importancia establecer el estado y el comportamiento de las variables inmersas en este tipo de modelos emergentes para reflejar tendencias en torno a la forma y la funcionalidad que a futuro podrían ser de gran utilidad y replicarse en condiciones similares.

\section{Caracterización}

Para el análisis de la problemática planteada se consideró la información obtenida por 12761 registros de estudiantes que tomaron espacios académicos comunes administrados por los Departamentos de Ciencias Básicas de dos Instituciones de Educación Superior (IEs) 
privadas de Bogotá — la Universitaria Agustiniana y la Universidad Santo Tomás- durante los periodos de 2019-2, 2020-1 y 2020-2.

El interés de considerar los periodos anteriormente descritos está dirigido a identificar las características en los procesos implementados para la evaluación que responde con los desempeños alcanzados por los estudiantes en los espacios académicos administrados por los Departamentos de Ciencias Básicas en el periodo anterior al aislamiento (2019-2), en el desarrollo del ciclo de transición (2020-1), y durante el aislamiento (2020-2).

Los criterios de exclusión se conceden a estudiantes que no cursan materias del componente básico, así como a los espacios académicos que no son comunes a las dos universidades. Cabe resaltar que se seleccionaron espacios académicos comunes de acuerdo con su contenido programático, mas no con el nombre particular de cada institución. Los espacios académicos seleccionados para nuestro estudio se describen en la Tabla 1.

Tabla 1: Espacios académicos de ciencias básicas comunes para la Universidad Santo Tomás y la Universitaria Agustiniana

\begin{tabular}{|l|c|c|c|}
\hline \multicolumn{1}{|c|}{ Asignatura } & Santo Tomás & Uniagustiniana & Total general \\
\hline Física mecánica & 612 & 350 & 962 \\
\hline Matemáticas II & 658 & 1412 & 2070 \\
\hline Álgebra lineal & 787 & 382 & 1169 \\
\hline Cálculo diferencial & 795 & 364 & 1159 \\
\hline Cálculo integral & 1004 & 383 & 1387 \\
\hline Cálculo vectorial & 647 & 246 & 893 \\
\hline Campos y ondas electromagnéticas & 75 & 33 & 108 \\
\hline Ecuaciones diferenciales & 546 & 301 & 847 \\
\hline Física, electricidad y magnetismo & 514 & 234 & 748 \\
\hline Lógica de programación & 579 & & 579 \\
\hline Matemáticas I & 682 & 1338 & 2020 \\
\hline Química general & 303 & 241 & 544 \\
\hline Termofluidos & 139 & 114 & 253 \\
\hline Variable compleja & 17 & 5 & 22 \\
\hline \multicolumn{1}{|c|}{ Total general } & 7358 & 5403 & 12761 \\
\hline
\end{tabular}

En particular, entre las variables a analizar se encuentran la universidad, el programa académico, el espacio académico - materia- y la nota definitiva. Como variable dependiente está la nota definitiva — variable cuantitativa en escala de medición de razón-, y en las variables explicativas tenemos la universidad — cualitativa nominal, con valores Uniagustiniana y Santo Tomás - y el programa académico — cualitativa nominal, cuyas categorías se distribuyen en los programas correspondientes a las Facultades de Ingeniería y Economía (Ciencias Económicas y Administrativas en la Uniagustiniana)—. 
La fuente principal de la información considera las bases de datos de reporte de notas en cada uno de los periodos descritos para la Universidad Santo Tomás y la Universitaria Agustiniana. El procesamiento de los datos se realiza por medio del software estadístico SPSS, una vez realizada la respectiva depuración de la información.

Partiendo de esto, la investigación descriptiva comprende la descripción, registro, análisis e interpretación de un fenómeno, y sus resultados parten de conclusiones fundamentales respecto a la funcionalidad o la interpretación correcta de la realidad del proceso o fenómeno estudiado. Teniendo en cuenta la metodología de la investigación descriptiva, las etapas que hicieron parte de la presente investigación fueron: (a) descripción del problema, (b) definición y formulación de hipótesis, (c) establecimiento del marco teórico que sustenta la investigación, (d) recolección de datos mediante el uso de instrumentos, (e) validación de los instrumentos, (f) descripción, análisis e interpretación de los datos, y (g) establecimiento de conclusiones.

\section{Resultados}

\section{Consideraciones sobre las herramientas de evaluación}

Las herramientas de evaluación validadas en los periodos objeto de estudio de la presente investigación tienen una relación directa con los resultados de aprendizaje obtenidos por los estudiantes. En este sentido, era necesario establecer un diagnóstico que correspondiera con los recursos implementados por los profesores en el desarrollo de la nueva realidad académica. Por esta razón, se consideró el uso de una encuesta realizada a los docentes de los departamentos de ciencias básicas de la Universidad Santo Tomás y de la Universitaria Agustiniana, para los cuales se generaron las siguientes reflexiones:

- Las herramientas más comunes para evaluar los resultados alcanzados por los estudiantes en las asignaturas de ciencias básicas incluyen evaluaciones escritas, cuestionarios de selección múltiple a través de plataformas virtuales, pruebas cortas escritas o a través de plataformas virtuales, talleres en grupo realizados en clase con la orientación del docente, trabajos en clase de laboratorio - toma de datos-, e informes de laboratorio.

- Para algunas asignaturas, las evaluaciones escritas que se realizaban en el semestre 2019-2 de manera presencial con preguntas abiertas migraron para el 2020-1 y 2020-2 a la forma virtual con preguntas de selección múltiple, respuesta calculada y preguntas aleatorias tomadas de un banco de preguntas o generadas mediante algún software. Para otras asignaturas, las evaluaciones escritas mantuvieron las preguntas abiertas, solo que 
ahora la entrega de la evaluación se hacía a través de alguna plataforma virtual o a través del correo electrónico.

- Para las asignaturas que tienen laboratorios, el trabajo presencial que se hacía hasta el 2019-2 y parte del 2020-1 tuvo que sustituirse por simulaciones o laboratorios virtuales con diferentes aplicativos disponibles en la Web, y en algunos casos se tuvo que trabajar con datos tomados en semestres anteriores para realizar un nuevo análisis. Sin embargo, las herramientas para la evaluación se mantuvieron, exigiendo la evidencia del trabajo en clase, la preparación de la práctica y el informe de laboratorio.

- Las pruebas escritas cortas y los talleres en clase se siguieron realizando en el semestre de transición, 2020-1 y en el semestre 2020-2, en plena pandemia, mediados por diferentes herramientas tecnológicas.

- No hubo cambios en cuanto a la distribución porcentual o peso asignado a cada una de las diferentes actividades propuestas para evaluar los resultados alcanzados por los estudiantes.

- Resaltamos un caso particular, que analizaremos más adelante, que se presentó con la asignatura Lógica de programación, ofertada a los programas de Ingeniería de la Universidad Santo Tomás. La particularidad es que las evaluaciones en los tres semestres bajo estudio no tuvieron cambios en cuanto a metodología, ya que todas ellas se realizaron a través de la plataforma Moodle.

\section{Resultados sobre el desempeño de los estudiantes}

Los análisis se llevaron a cabo sobre 12761 registros de estudiantes, de los cuales el $42.3 \%$ pertenecía a la Universitaria Agustiniana y el 57.7 \% a la Universidad Santo Tomás. La participación en los tres periodos fue de 35 \% para el 2019-2, 37 \% para el 2020-1 y $28.1 \%$ para el 2020-2. Los programas con mayor participación en el estudio fueron Ingeniería Industrial con un 17.4 \%, seguido por Negocios Internacionales con 12 \% e Ingeniería Civil con un 10 \%; los demás programas tuvieron un porcentaje menor al 10 \% (véase Figura 1). 
Figura 1: Distribución de registros por materia

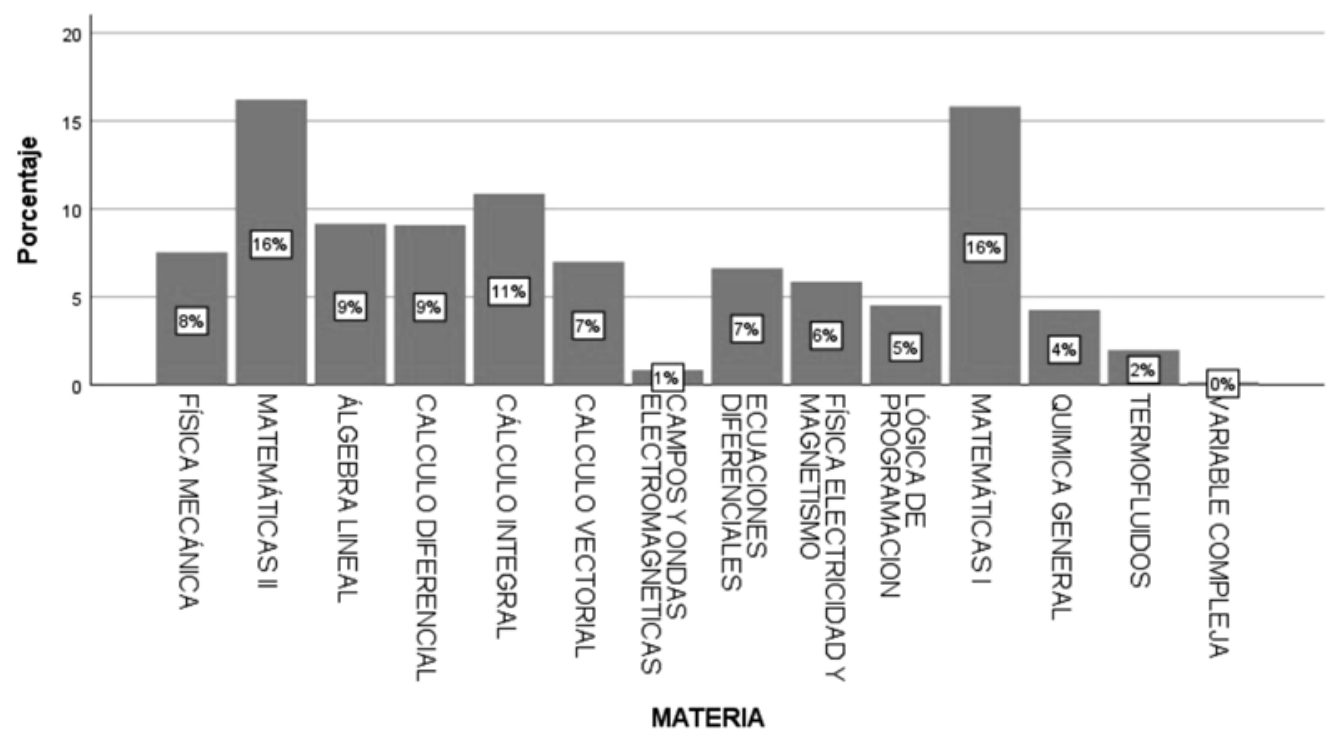

De acuerdo con los registros por espacio académico — materia—, se observa que tanto Matemáticas I como Matemáticas II tienen mayor cantidad de información, con un 16 \% para cada una de ellas, seguidas por Cálculo integral con un 11 \%, Cálculo diferencial con un $9 \%$, y Álgebra lineal con un $9 \%$. Las materias con menor porcentaje de participación fueron las correspondientes a las áreas de Física, con Termofluidos (2\%) y Campos y ondas (1 \%), y Química, con Química general (4 \%). Para los cursos más avanzados se encuentra Variable compleja con menos del $1 \%$ en los registros analizados.

Por otra parte, en la Tabla 2 se resumen los estadísticos para la variable definitiva, donde se observa que la nota promedio de los estudiantes de las dos universidades en los tres periodos fue de 3.6, con una desviación de 0.887 unidades respecto a la media. Por otro lado, en la Figura 2 se muestra la evolución del promedio en cada uno de los periodos para las dos universidades. Se puede observar que existe una tendencia creciente en la nota definitiva promedio del 2019-2 al 2020-2, lo cual sustenta la hipótesis del cambio de la evaluación reflejada en la evolución del promedio de la nota en la situación actual. 
Tabla 2: Estadísticos descriptivos para la nota definitiva

\begin{tabular}{|c|c|c|}
\hline \multicolumn{2}{|r|}{ Definitiva } & Valor \\
\hline \multirow{2}{*}{$\mathrm{N}$} & Válido & 12761 \\
\hline & Perdidos & 0 \\
\hline \multicolumn{2}{|c|}{ Media } & 3.6435 \\
\hline \multicolumn{2}{|c|}{$\mathrm{DE}$} & 0.87738 \\
\hline \multicolumn{2}{|c|}{ Asimetría } & -1.858 \\
\hline \multicolumn{2}{|c|}{ Error estándar de asimetría } & 0.022 \\
\hline \multicolumn{2}{|c|}{ Curtosis } & 5.669 \\
\hline \multicolumn{2}{|c|}{ Error estándar de curtosis } & 0.043 \\
\hline \multicolumn{2}{|c|}{ Mínimo } & 0.00 \\
\hline \multicolumn{2}{|c|}{ Máximo } & 5.00 \\
\hline
\end{tabular}

Figura 2: Distribución de registros por materia

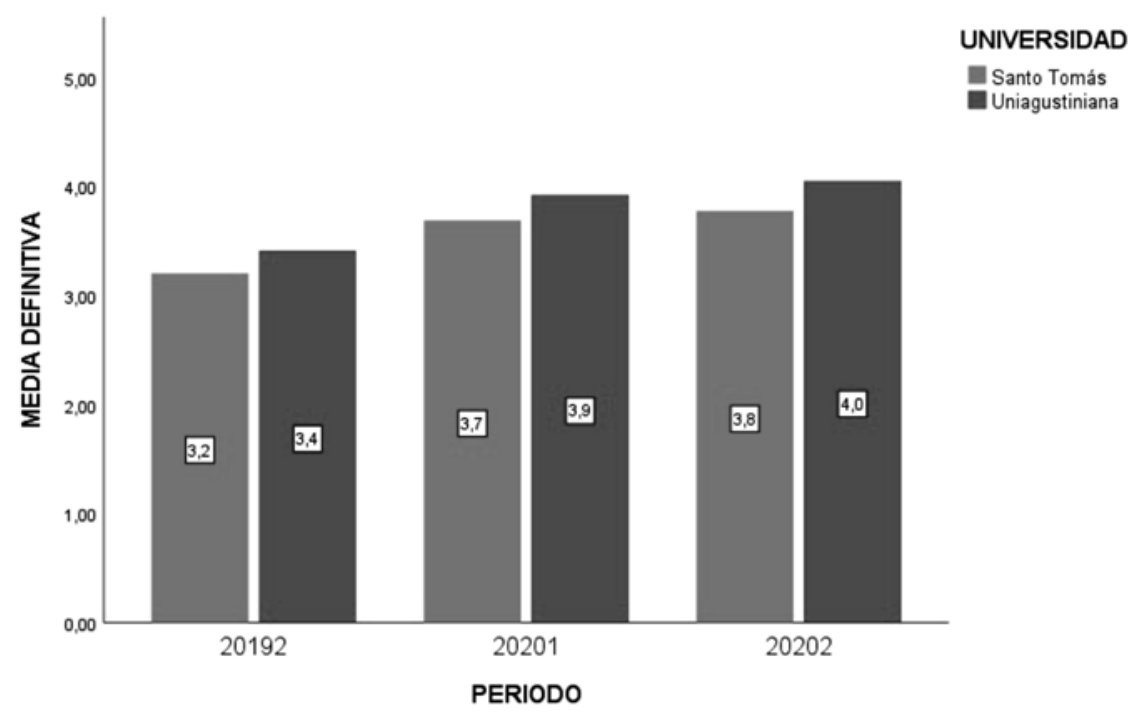

Ahora bien, de acuerdo con los estadísticos descriptivos que se presentan la Tabla 3 para la nota definitiva, se observa que la materia que mayor nota promedio tuvo fue Campos y ondas electromagnéticas, con 3.88 de valor medio y una desviación estándar de 0.61 , seguida por Cálculo vectorial, con una media de 3.86 y una desviación estándar de 0.80, y Matemáticas II, con un valor medio de 3.75 y una desviación estándar de 0.82 . Por su parte, los cursos con menor promedio en la definitiva fueron Física mecánica, con 3.44 en valor medio y una desviación de 0.73 , Variable compleja, con una media de 3.54 y una desviación de 0.50 , y Álgebra lineal, con un promedio de 3.58 y una desviación de 0.99 . 
Tabla 3: Estadísticos descriptivos para la nota definitiva discriminada por materia

\begin{tabular}{|l|c|c|c|c|c|}
\hline \multicolumn{1}{|c|}{ Asignatura } & $\mathrm{N}$ & Mínimo & Máximo & Media & $\mathrm{DE}$ \\
\hline Física mecánica & 962 & 0.00 & 5.00 & 3.4449 & 0.73745 \\
\hline Matemáticas II & 2070 & 0.00 & 5.00 & 3.7439 & 0.82577 \\
\hline Álgebra lineal & 1169 & 0.00 & 5.00 & 3.5881 & 0.99841 \\
\hline Cálculo diferencial & 1159 & 0.00 & 5.00 & 3.4610 & 1.08048 \\
\hline Cálculo integral & 1387 & 0.00 & 5.00 & 3.6978 & 0.88499 \\
\hline Cálculo vectorial & 893 & 0.00 & 5.00 & 3.8669 & 0.80640 \\
\hline Campos y ondas electromagnéticas & 108 & 1.60 & 5.00 & 3.8870 & 0.61509 \\
\hline Ecuaciones diferenciales & 847 & 0.00 & 5.00 & 3.6764 & 0.68571 \\
\hline Física, electricidad y magnetismo & 748 & 0.00 & 5.00 & 3.6847 & 0.64969 \\
\hline Lógica de programación & 579 & 0.00 & 5.00 & 3.6234 & 0.74983 \\
\hline Matemáticas I & 2020 & 0.00 & 5.00 & 3.6824 & 0.96798 \\
\hline Química general & 544 & 0.00 & 4.70 & 3.3479 & 0.88485 \\
\hline Termofluidos & 253 & 0.00 & 4.80 & 3.6292 & 0.62102 \\
\hline Variable compleja & 22 & 3.00 & 4.90 & 3.5436 & 0.50152 \\
\hline
\end{tabular}

Por otra parte, atendiendo que la nota reprobatoria en las dos universidades corresponde a un puntaje menor a 3.0 puntos finales, se encuentra que el porcentaje de pérdida general fue menor para el periodo 2020-1 (6 \%), con un aumento al $7 \%$ en el periodo 2020-2, pero no mayor al 2019-2 (14 \%) (véase Figura 3), situación que se sustenta bajo el cambio de las estrategias de evaluación en los periodos comprendidos: antes, en transición y durante el aislamiento.

Figura 3: Distribución de pérdida por periodo

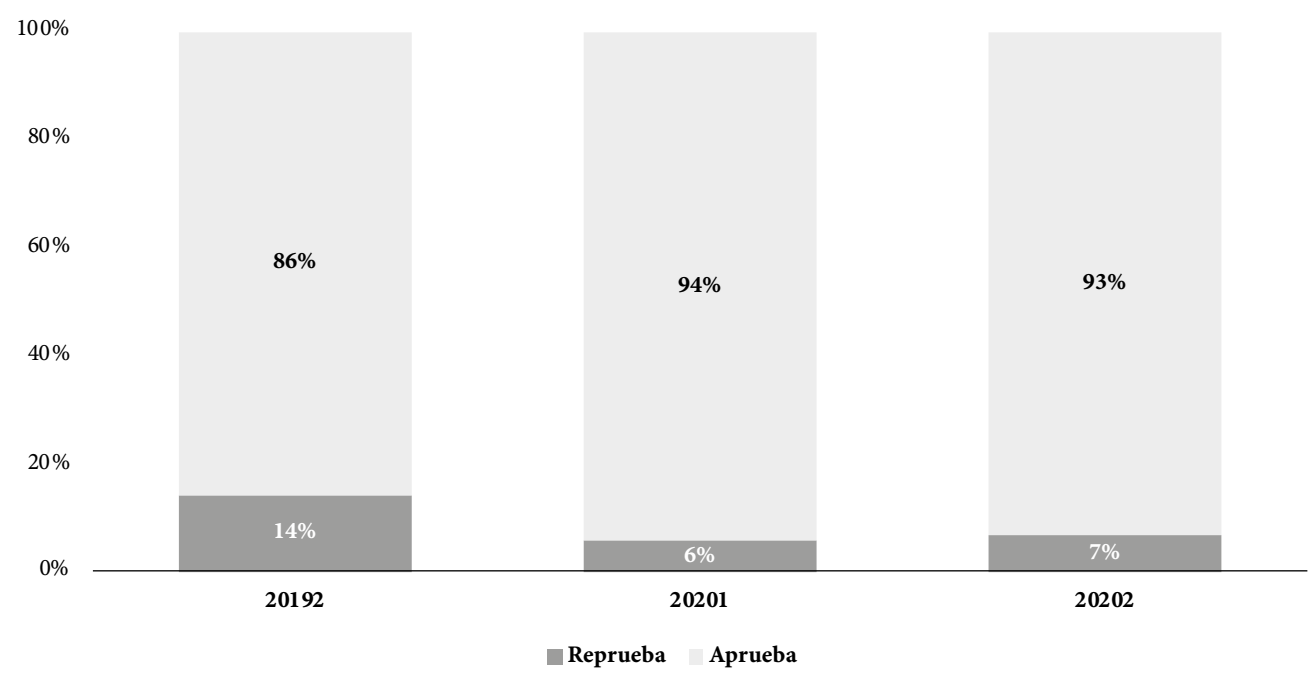


En cuanto a los programas con mayor pérdida (véase Figura 4) se encuentra Tecnología en Desarrollo de Software de la Facultad de Ingeniería de la Universitaria Agustiniana — con un 19 \% de reprobación en los tres periodos_, seguida por Ingeniería Ambiental de la Universidad Santo Tomás y Tecnología de Gastronomía de la Universitaria Agustiniana —ambas con $14 \%$ de pérdida en los tres períodos—. El menor porcentaje de pérdida se halló en el programa de Hotelería y Turismo de la Universitaria Agustiniana.

Figura 4: Distribución de pérdida por programa

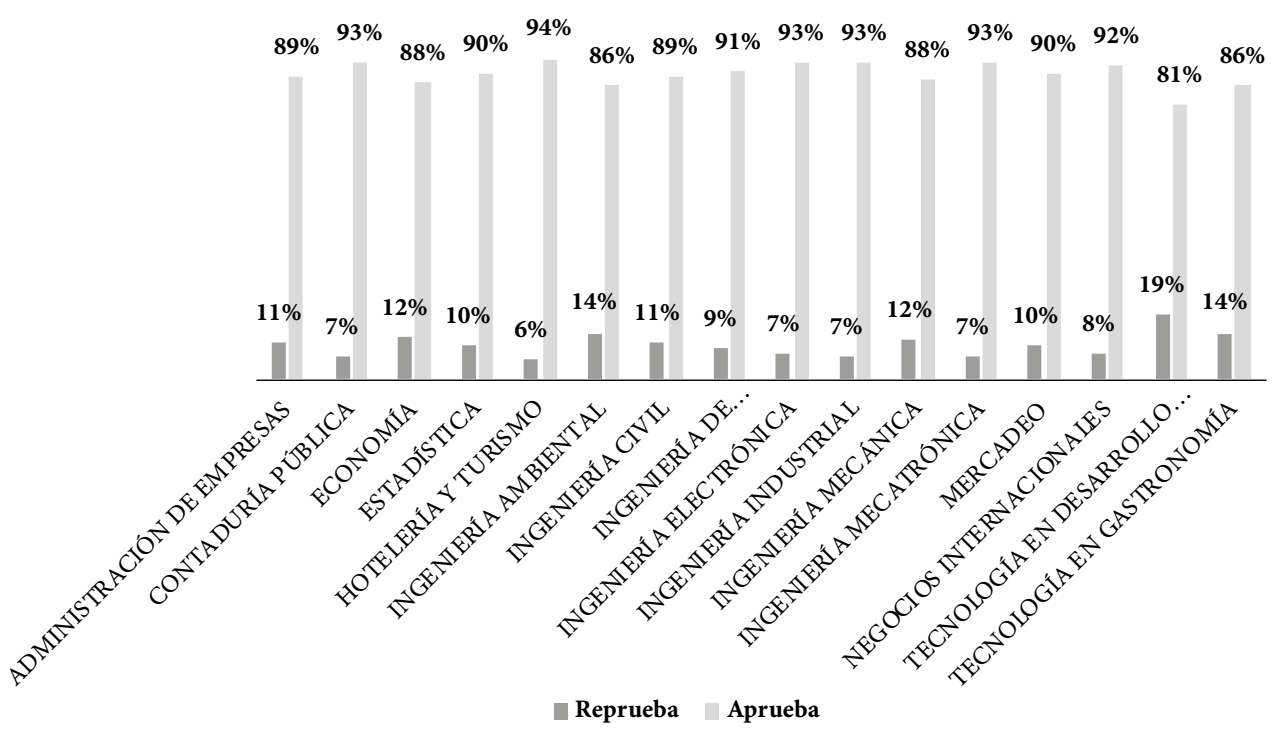

De acuerdo con los resultados por materia, se observa que las materias con mayor porcentaje de pérdida en los tres periodos analizados fueron Cálculo diferencial (15\%), Química general (14\%) y Física mecánica (12 \%), mientras que las materias con menor pérdida en los tres periodos fueron Variable compleja (menos del $1 \%$ ), Campos y ondas (2 \%) y Termofluidos (4\%) (véase Figura 5). 
Figura 5: Distribución de pérdida por materia

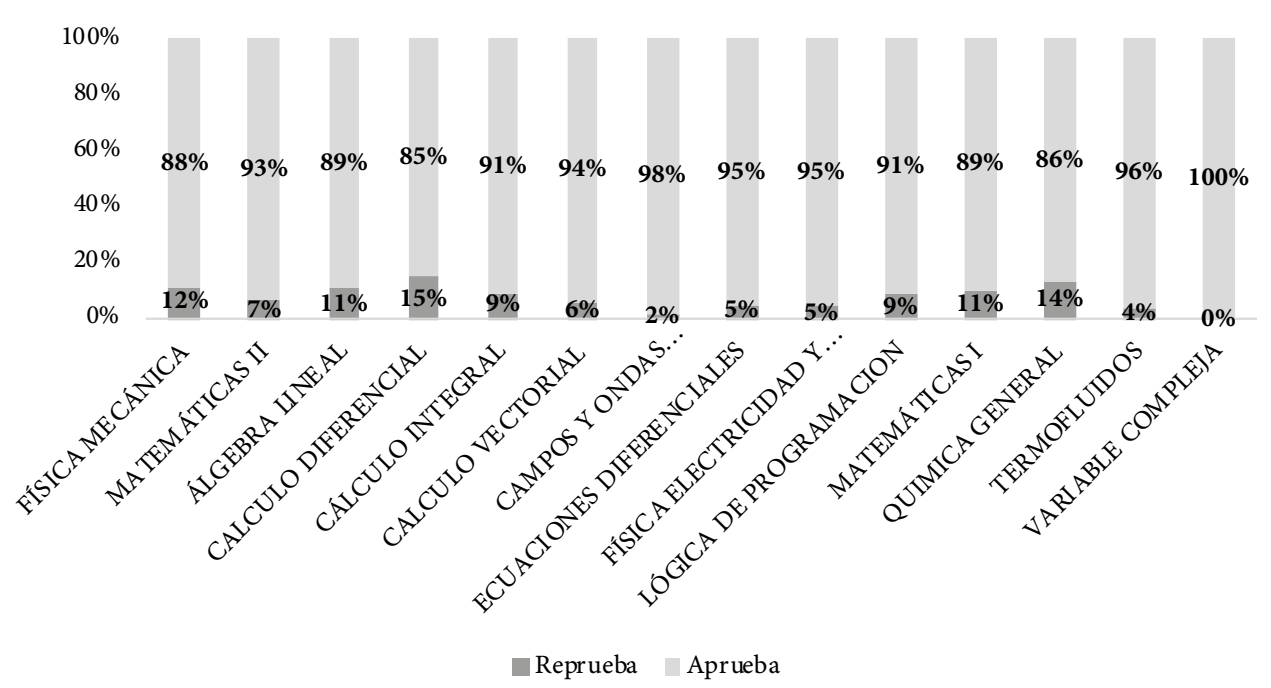

Una vez identificado el desempeño en los tres periodos en términos generales y discriminados por materias, al igual que la reprobación para los programas y en cada una de las materias comunes en las dos universidades, se hace necesario identificar si existen diferencias significativas en la calificación en los tres periodos analizados, con el fin de responder a las diferencias existentes en la evaluación antes, en el periodo de transición y durante la pandemia, con los cambios en el desempeño de los estudiantes — atendiendo a las herramientas de enseñanza y evaluación de los aprendizajes en las clases remotas con apoyo de la tecnología-. De acuerdo con lo anterior, se realizó la prueba no paramétrica Kruskall-Wallis de diferencia de grupos independientes como alternativa a la prueba paramétrica ANOva (mostrada en la Tabla 5), tras haber identificado la no normalidad en los grupos — con una significancia de 0.000 menor a 0.005- (véase Tabla 4), ya que, de acuerdo con Flores-Ruiz et al. (2017),

Cuando la distribución de datos cuantitativos no sigue una distribución normal también hay diferentes pruebas estadísticas con las que se comparan las medianas [...] En el caso de 3 o más grupos independientes se debe utilizar la prueba de Kruskal-Wallis (la cual es equivalente a ANova de una vía). (p. 369) 
Tabla 4: Prueba de normalidad por periodo

\begin{tabular}{|c|c|c|c|}
\hline & Periodo & $\mathrm{N}$ & Rango promedio \\
\hline \multirow{4}{*}{ Definitiva } & $2019-2$ & 4454 & 4624.33 \\
\cline { 2 - 4 } & $2020-1$ & 4724 & 7132.89 \\
\cline { 2 - 4 } & $2020-2$ & 3583 & 7573.37 \\
\cline { 2 - 4 } & Total & 12761 & \\
\hline
\end{tabular}

Tabla 5: Prueba no paramétrica de diferencia de medias Kruskal-Wallis

\begin{tabular}{|c|c|}
\hline & Definitiva \\
\hline H de Kruskal-Wallis & 1587.150 \\
\hline gl & 2 \\
\hline Sig. asintótica & 0.000 \\
\hline
\end{tabular}

Nota. Prueba de Kruskal Wallis. Variable de agrupación: Periodo.

En este punto, los resultados indican, con un nivel de significancia de 0.05 y un $p$-valor de 0.000 menor que la significancia, que se rechaza la hipótesis de igualdad de promedios, por lo que se puede decir que el promedio de definitiva es diferente en al menos uno de los periodos. Es así como, comparando parejas de periodos, se confirman diferencias dos por dos, mostrando que el promedio de la definitiva es diferente en el 2019-2, 2020-1 y 2020-2.

Por último, y con el objetivo de identificar si existe relación entre la pérdida y el periodo académico, se realizó una prueba Chi-cuadrado de dependencia (véase Tabla 6), atendiendo a que, dadas las diferencias anteriores, se puede intuir que el desempeño responde al periodo en el cual se cursó la materia.

Tabla 6: Prueba de chi-cuadrado de independencia

\begin{tabular}{|c|c|c|c|}
\hline & Valor & DF & Significación asintótica (bilateral) \\
\hline Chi-cuadrado de Pearson & $224.865^{*}$ & 2 & 0.00 \\
\hline Razón de verosimilitud & 214.8352999 & 2 & 0.00 \\
\hline Asociación lineal por lineal & 146.589 & 1 & 0.000 \\
\hline N de casos válidos & 12761 & & \\
\hline
\end{tabular}

Nota. * 0 casillas $(0.0 \%)$ han esperado un recuento menor que 5. El recuento mínimo.

De acuerdo con los resultados, y con un valor $p$ de 0.000 , menor que la significancia (0.005), se rechaza la hipótesis de independencia, por lo que estadísticamente se puede comprobar que la condición de reprobar la materia está relacionada con el periodo académico. 


\section{Discusión}

Las implicaciones de la pandemia han afectado todos los sectores que componen la realidad establecida por este mundo globalizado, sin dejar de lado la educación en los entornos propios de la formación profesional universitaria.

En este sentido, y en correspondencia con la hipótesis planteada en el presente trabajo, los procesos de evaluación enmarcados en modalidad remota muestran un crecimiento significativo en valoraciones positivas de aprobación, considerando los resultados para los desempeños definidos en un espacio académico, que no solo afectan los registros de la evaluación para un grupo de estudiantes, sino que validan un incremento considerable de los estándares de aprobación en las instituciones para los periodos de 2019-2 a 2020-2. En ese orden de ideas, se resaltan las materias Campos y ondas, Cálculo vectorial y Matemáticas II como aquellas con mayor nota promedio, mientras que con menor nota promedio se encuentran Física mecánica, Variable compleja y Álgebra lineal.

Asimismo, es posible afirmar que los procesos remotos de evaluación con la utilización de estrategias virtuales propias de los insumos tecnológicos han permitido un incremento en los resultados obtenidos para los desempeños definidos por los espacios académicos relacionados con el estudio, considerando que el porcentaje de pérdida para el periodo definido corresponde a un $6 \%$, frente a un porcentaje de dos dígitos, habitual en los procesos académicos anteriores a este periodo. No obstante, se ha revelado que no todos los estudiantes asumieron con responsabilidad su rol.

De manera general, los resultados obtenidos en este estudio muestran un incremento en la nota definitiva promedio para el periodo 2020-1 con respecto al 2019-2, tal vez un incremento esperado, toda vez que los procesos de evaluación fueron ajustados con el propósito de responder a la nueva realidad sin perder el desarrollo de los objetivos de aprendizaje definidos por los espacios académicos y considerando la necesidad de continuar con un proceso evaluativo formativo que se ajustara con las necesidades particulares de los estudiantes frente al saber disciplinar y la interacción con su aprendizaje.

Por su parte, el incremento en la nota definitiva promedio para el periodo 2020-2 con respecto al 2020-1 puede entenderse, en parte, porque se consolidaron estrategias ideadas por los docentes para el seguimiento y la evaluación que posibilitaron un seguimiento ajustado a un conjunto de lineamientos, con lo cual fue posible la exigencia académica, teniendo en cuenta que los estudiantes matriculados en este periodo de aislamiento ya disponían de los medios para ser parte de la presencialidad mediada por la tecnología. 
Así como los docentes ganamos experiencia en el manejo de diferentes herramientas y ayudas tecnológicas, lo propio hicieron los estudiantes. Infortunadamente, el plagio se ha convertido en un protagonista de los procesos evaluativos bajo la modalidad virtual, y a su vez ha agudizado el ingenio de los docentes para tratar de evitarlo. Por esta razón, se pone en tela de juicio si es verdad que el incremento en la nota definitiva alcanzada por los estudiantes refleja un incremento en las destrezas adquiridas frente a los resultados de aprendizaje definidos para un grupo de competencias. En este sentido, habría que esperar el retorno completo a la presencialidad para evaluar si la experiencia adquirida en el manejo de las herramientas virtuales puede contribuir positivamente a la modernización y dinamización de la educación, y así al desempeño de los estudiantes.

\section{Conclusiones}

Algunas de las conclusiones generales encontradas en este estudio y sus análisis estadísticos pueden ser consideradas a partir de las siguientes afirmaciones:

- El porcentaje de pérdida general fue menor para el periodo 2020-1, con un 6 \%, y tuvo un aumento al 7 \% en el periodo 2020-2, pero no mayor al 2019-2 (14 \%), situación que se sustenta bajo el cambio de las estrategias de evaluación en los periodos comprendidos: antes, en transición y durante el aislamiento.

- Los programas con mayor pérdida fueron Tecnología en Desarrollo de Software de la Facultad de Ingeniería de la Universitaria Agustiniana, seguido por Ingeniería Ambiental de la Universidad Santo Tomás y Tecnología de Gastronomía de la Universitaria Agustiniana.

- Las materias con mayor porcentaje de pérdida fueron Cálculo diferencial (15 \%), Química general y Física mecánica. Las materias con menor pérdida en los tres periodos fueron Variable compleja, Campos y ondas y Termofluidos.

- $\quad$ El porcentaje de pérdida fue menor para el periodo 2020-1 —el periodo de transición-, por lo que se podría afirmar que la evaluación fue menos exigente por la adopción del uso de las tecnologías de la información para desarrollar las clases y evaluar el curso. A pesar de que el porcentaje de reprobación aumentó en el periodo 2020-2 en comparación con el periodo anterior, en un contexto en el que el manejo de las herramientas y uso de recursos para el desarrollo de las clases se perfeccionó, el porcentaje no fue mayor al periodo inicial del 2019-1, y el promedio no descendió, sino que, por el contrario, continuó una tendencia en aumento. 
- Resultados estadísticos mostraron que, en todos los periodos desde que inició la pandemia hasta finales del 2020, los desempeños de los estudiantes medidos a partir de la calificación definitiva al final del curso son diferentes. Esto puede deberse, en cierta medida, a los cambios en la evaluación, el perfeccionamiento en el uso de herramientas tecnológicas y la facilidad en el acceso a la información.

- Existe relación entre la reprobación y el periodo académico, lo que significa que el mayor porcentaje de pérdida en el 2019-2 responde al periodo prepandemia. Este mismo disminuyó para el periodo 2020-1, siendo un periodo de cambio y reajustes de los procesos académicos y evaluativos, así como de acomodación; y, finalmente, la reprobación para el 2020-2 aumentó, pero el crecimiento no fue significativo, lo que podría asociarse con la adaptación por parte de los docentes y de los estudiantes a las nuevas estrategias de orientación, seguimiento y evaluación implementadas.

\section{Referencias}

Camacho, M. (2020, mayo 26). La evaluación de los aprendizajes en tiempos de pandemia. El blog de Studia XXI Universidad: Una conversación pública sobre la universidad. https://www. universidadsi.es/evaluacion-online-covid19/

Flores-Ruiz, E., Miranda-Novales, M. G., Villasís-Keever, M. Á. (2017). El protocolo de investigación VI: cómo elegir la prueba estadística adecuada. Estadística inferencial. Revista Alergia México, 64 (3), 364-370. http://www.scielo.org.mx/pdf/ram/v64n3/2448-9190-ram-64-03-0364.pdf

Luthra, P. (2020) Una ocasión para reinventar la escuela. Correo de la UNESCO: un solo mundo, voces múltiples. https://es.unesco.org/courier/2020-3/ocasion-reinventar-escuela

Nevo, D. (1997). Evaluación basada en el centro. Un diálogo para la mejora educativa. Mensajero.

Sabino, C. (2014). El proceso de investigación. Editorial Episteme.

Trujillo, F. , Navas, M., Segura, A., \& Jiménez, M. (2020). Escenarios de Evaluación en el Contexto de la Pandemia por la COVID-19: La Opinión del Profesorado. SantillanaLab.

Vicenti, I. (2020). La educación superior en tiempos de COVID-19: Aportes de la Segunda Reunión del Diálogo Virtual con Rectores de Universidades Líderes de América Latina. Banco Interamericano de Desarrollo. https://publications.iadb.org/publications/spanish/document/La-educacion-superior-entiempos-de-COVID-19-Aportes-de-la-Segunda-Reunion-del-Di\%C3\%A1logo-Virtual-con-Rectores-deUniversidades-Lideres-de-America-Latina.pdf 